ARTICLE

https://doi.org/10.1038/s41467-019-09502-5

\title{
In-situ electron microscopy mapping of an order-disorder transition in a superionic conductor
}

\author{
Jaeyoung Heo', Daniel Dumett Torres², Progna Banerjee ${ }^{3}$ \& Prashant K. Jain (1) 2,3,4,5
}

Solid-solid phase transitions are processes ripe for the discovery of correlated atomic motion in crystals. Here, we monitor an order-disorder transition in real-time in nanoparticles of the super-ionic solid, $\mathrm{Cu}_{2-x} \mathrm{Se}$. The use of in-situ high-resolution transmission electron microscopy allows the spatiotemporal evolution of the phase transition within a single nanoparticle to be monitored at the atomic level. The high spatial resolution reveals that cation disorder is nucleated at low co-ordination, high energy sites of the nanoparticle where cationic vacancy layers intersect with surface facets. Time-dependent evolution of the reciprocal lattice of individual nanoparticles shows that the initiation of cation disorder is accompanied by a $\sim 3 \%$ compression of the anionic lattice, establishing a correlation between these two structural features of the lattice. The spatiotemporal insights gained here advance understanding of order-disorder transitions, ionic structure and transport, and the role of nanoparticle surfaces in phase transitions.

\footnotetext{
${ }^{1}$ Department of Materials Science \& Engineering, University of Illinois at Urbana-Champaign, Urbana, IL 61801, USA. ${ }^{2}$ Department of Chemistry, University of Illinois at Urbana-Champaign, Urbana, IL 61801, USA. ${ }^{3}$ Department of Physics, University of Illinois at Urbana-Champaign, Urbana, IL 61801, USA. ${ }^{4}$ Materials Research Laboratory, University of Illinois at Urbana-Champaign, Urbana, IL 61801, USA. ${ }^{5}$ Beckman Institute of Advanced Science and Technology, University of Illinois at Urbana-Champaign, Urbana, IL 61801, USA. Correspondence and requests for materials should be addressed to P.K.J. (email: jain@illinois.edu)
} 
T he advent of in-situ high-resolution transmission electron microscopy (HRTEM) has made available a tool for monitoring solid-state phase transitions and elucidating their underlying atomistic dynamics ${ }^{1-4}$. In-situ HRTEM has been exploited to gain a deeper understanding of the crystallization and growth of nanoparticles ${ }^{5,6}$ and post-synthetic transformations of nanoparticles, such as hydrogenation ${ }^{7}$. Here, we use insitu HRTEM to follow an order-disorder phase transition with atomic resolution.

The transition we investigate is central to fast-ion conducting solids, which have potential as solid electrolytes for Li-ion batteries $^{8-15}$. Solids in this class are crystalline at room temperature; but at elevated temperatures, they exhibit a disordered cationic sub-lattice with high cation mobility. As our model system, we choose cuprous selenide $\left(\mathrm{Cu}_{2} \mathrm{Se}\right.$ or $\left.\mathrm{Cu}_{2-x} \mathrm{Se}\right)$, a common fast-ion conductor ${ }^{16-22}$. The choice was motivated by the structural features of $\mathrm{Cu}_{2} \mathrm{Se}$. A fraction of the $\mathrm{Cu}^{+}$in $\mathrm{Cu}_{2} \mathrm{Se}$ is displaced from the ideal tetrahedral sites within the near-face-centered cubic $\mathrm{Se}^{2-}$ sub-lattice cage ${ }^{21,23-25}$. The resulting tetrahedral-site $\mathrm{Cu}-$ vacancies order to form a super-lattice ${ }^{16,19,21,26-28}$ with a characteristic inter-vacancy-layer spacing of $\sim 0.68 \mathrm{~nm}$. But, at temperatures greater than $\sim 140{ }^{\circ} \mathrm{C}$ in bulk $\mathrm{Cu}_{2} \mathrm{Se}$, the $\mathrm{Cu}^{+}$becomes mobile over multiple types of interstitial sites and the vacancy ordered (VO) structure ${ }^{23,25,26}$ is lost. The resulting superionic (SI) phase of $\mathrm{Cu}_{2} \mathrm{Se}$ exhibits fast-ion conduction ${ }^{19,25}$. We are motivated to study the temperature-induced transition from the ordered phase to the SI phase, because newer understanding can lead to strategies for enhancing ion transport and reducing the temperature needed for achieving fast-ion conduction and bringing it closer to the operating temperature range of $\mathrm{Li}$-ion batteries.

Using in-situ HRTEM, we watch the VO-to-SI transition within a single $\mathrm{Cu}_{2-x} \mathrm{Se}$ nanoparticle, providing us a unprecedented look at the spatiotemporal progression of the phase transition. We identify the precise atomic site where cation disordering is initiated and uncover correlated structural motions involved in the process.

\section{Results}

Nanoparticles with an order-disorder transition. We filmed using in-situ HRTEM individual $\mathrm{Cu}_{2-\mathrm{x}} \mathrm{Se}$ nanoparticles as they underwent a transition from the VO phase to the SI phase (Fig. 1, Supplementary Movies 1 and 2). The nanoparticles have a hexagonally shaped cross-section with a mean size of $\sim 22 \mathrm{~nm}$ measured along the diagonals of the hexagon (Supplementary Fig. 1). At this relatively large size, the hexagonal nanoparticles (HNPs) have a phase transition temperature of $\sim 140^{\circ} \mathrm{C}$ similar to that of the bulk solid ${ }^{23}$. But, unlike the bulk solid, surface facets comprise an important feature of the HNPs. We refer to the HNPs using the sub-stoichiometric $\mathrm{Cu}_{2-\mathrm{x}} \mathrm{Se}$ formula simply to account for a small amount of air oxidation that the HNPs undergo in the course of their transfer to the HRTEM instrument. However, the small degree of sub-stoichiometry, $x$, does not cause any deviation in the lattice structure of these HNPs relative to that of stoichiometric $\mathrm{Cu}_{2} \mathrm{Se}$. Prior to any transition, (Fig. 1), the HRTEM images of the HNPs show the characteristic lattice fringe pattern of low-temperature $\mathrm{Cu}_{2} \mathrm{Se}$. A lattice fringe pattern with a period of $\sim 0.68 \mathrm{~nm}$ is observed along the [111 $]_{\mathrm{c}}$ crystallographic axis of the pseudo-cubic (c) unit cell. This pattern is a manifestation of a $\mathrm{Cu}$-vacancy super-lattice formed by the arrangement of $\mathrm{Cu}$ vacancies once every fourth $\mathrm{Cu}^{+}$layer along $[111]_{c}{ }^{29}$.

In-situ imaging of order-disorder phase transition. The temperature-dependent phase transition in an HNP was induced by the electron beam, which heats up the $\mathrm{HNP}^{3,30}$. From the estimates of the local temperature achieved under the electron beam irradiation conditions of our studies (see Supplementary Note), it is plausible for beam-induced heating to raise the temperature of an HNP to a value above the phase transition temperature of $\sim 140^{\circ} \mathrm{C}$. As the beam-induced phase transition progressed, a high-resolution movie of the HNP at 1 frame per $s$ was acquired. Select snapshots from such a movie of a representative HNP are shown in Fig. 1a. As shown by the example, the HNPs start in the VO phase and eventually transition to the SI phase under the focused electron beam. The formation of the SI phase is seen as a loss of the ordered Cu-vacancy super-lattice of $\sim 0.68 \mathrm{~nm}$ periodicity, as also confirmed by the fast-Fourier transforms (FFTs) of VO and SI regions in the real-space image (Fig. 1b). In the SI phase, lattice fringes are observed with a periodicity of $\sim 0.33 \mathrm{~nm}$, which is the characteristic $\mathrm{Se}^{2-}-\mathrm{Se}^{2-}$ interlayer spacing along $[111]_{c}^{16,26}$. In other words, the $\mathrm{Cu}^{+}$sublattice, being disordered, does not contribute to the lattice pattern.

Precise site for nucleation of cation disorder. As seen from Fig. 1a, at intermediate stages in the transition, domains of the SI and VO phases co-exist in the HNP. With increasing time, the SI domain grows in size and the VO domain shrinks until the HNP is fully in the SI phase. We used the characteristic lattice fringe spacing $\mathrm{d}_{[111] \mathrm{c}}$ to identify the portions of the HNP that were in the VO $\left(\mathrm{d}_{[111] \mathrm{c}} \approx 0.68 \mathrm{~nm}\right)$ and SI $\left(\mathrm{d}_{[111] \mathrm{c}} \approx 0.33 \mathrm{~nm}\right)$ phases. In this manner, we were able to obtain spatial maps (Fig. 1c) and time-trajectories (Supplementary Fig. 2) of the growth of the SI phase in a single HNP as a function of time.

We found that the phase transition was invariably initiated at the surface of the HNP, specifically at a vertex site (Fig. 1c and Supplementary Fig. 3), a feature observed for 14 of $15 \mathrm{Cu}_{2-x} \mathrm{Se}$ HNPs were studied. A similar phenomenon has been observed for hydrogen intercalation in faceted $\alpha$-palladium hydride nanoparticles $^{31}$. Vertex sites on a nanoparticle surface have a lower atomic co-ordination than other lattice sites, resulting in a higher local surface energy. As a result, the energy barrier for nucleation of the SI phase can be considerably lower at such sites. There is an alternate possibility: due to the smaller mass thickness at the vertex sites, the electron beam effect is pronounced at these locations, resulting in preferential nucleation at these sites. If this were the case, then nucleation would have been observed at any one of the six vertices of the HNPs. However, as seen for three representative HNPs in Fig. 1c, the transition nucleated at only the specific vertex, or pair of vertices, which lie at the intersection of the $(\overline{1} 11)_{c}$ and $(100)_{c}$ surface facets (Supplementary Fig. 4). There is a possible reason why this specific pair of vertices is the favored location for nucleation of cation disorder. These vertices are located normal to the direction along which $\mathrm{Cu}$-vacancy planes are oriented. The vacancy planes intersect these vertices, reducing the local coordination number and increasing surface energy even further. In essence, we find that the phase transition is initiated at the site of confluence of surface atoms and $\mathrm{Cu}$ vacancies, where atomic co-ordination is likely to be the weakest. The spatial manner in which the SI phase nucleates and grows across the $\mathrm{Cu}_{2-\mathrm{x}} \mathrm{Se}$ HNP is depicted by the schematic in Fig. $2 \mathrm{a}$.

Electron beam effects involved in the phase transition. Since the electron beam is employed not only for imaging but also for inducing the transition, we characterized electron beam effects in more detail and examined the role of multiple effects. In general, electron beam irradiation can influence a specimen in two ways: via elastic scattering effects such as knock-on damage ${ }^{30}$ and inelastic scattering effects such as specimen heating and electrostatic charging 32,33 . 
a
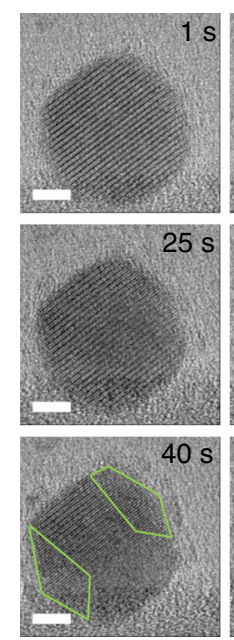

$0 \mathrm{~s}$
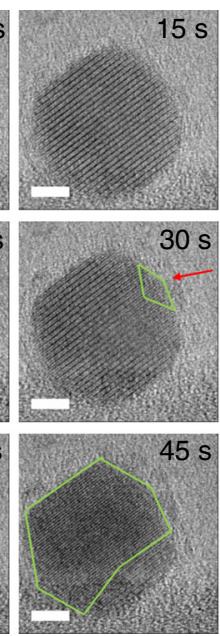

C
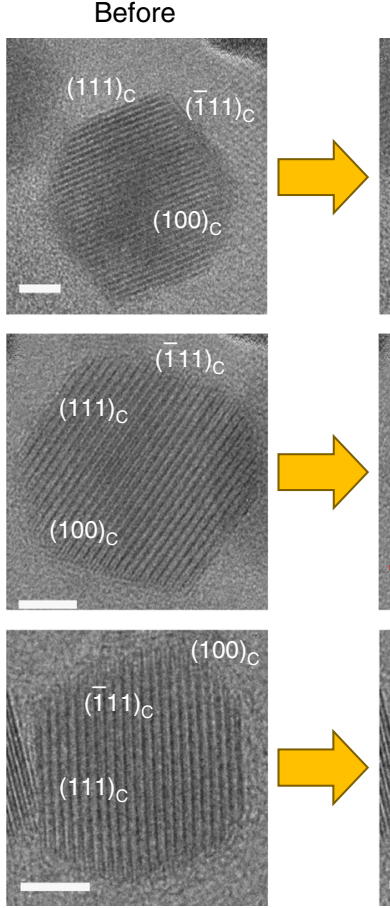
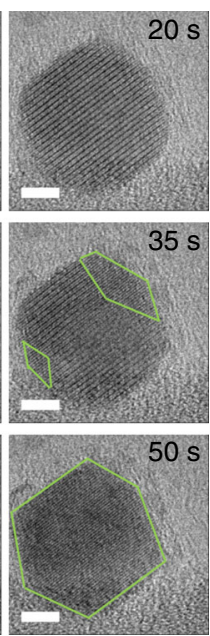

Nuclation

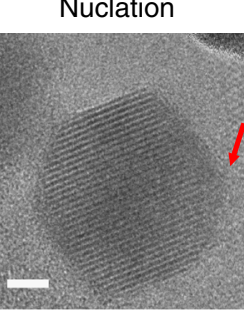

b
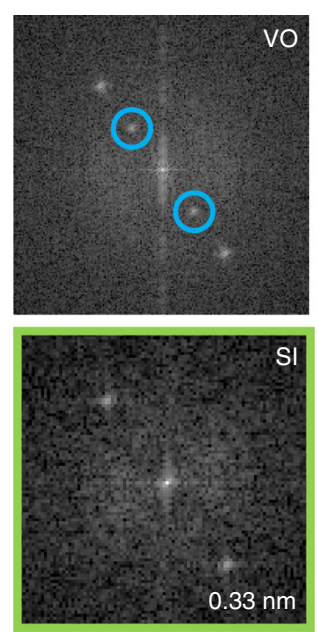

After
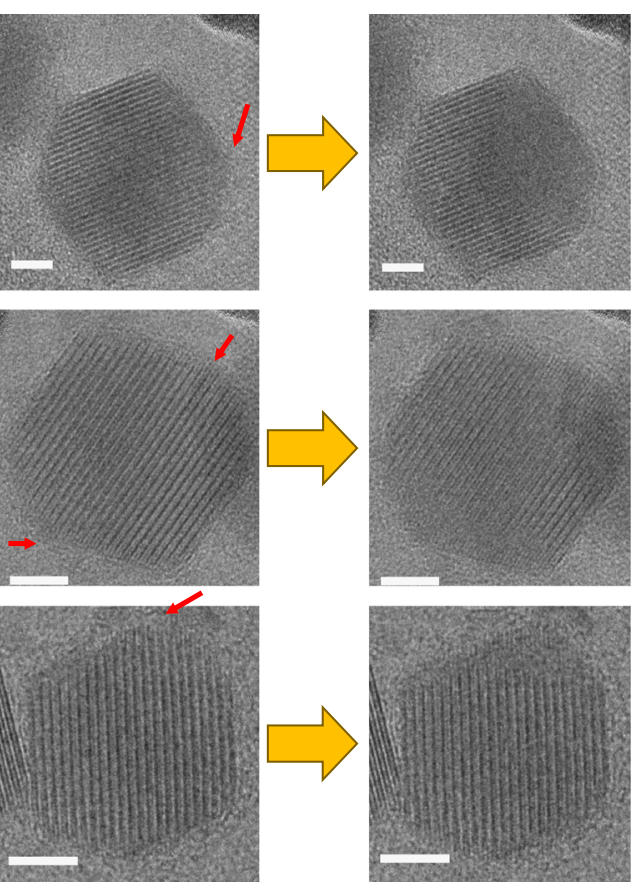

Fig. 1 HRTEM imaging of the order-disorder phase transition in $\mathrm{Cu}_{2-x} \mathrm{Se}$ HNPs. a Selected movie frames from continuous HRTEM imaging of an HNP undergoing a transition from the vacancy-ordered (VO) phase to the superionic (SI) phase. The time relative to the start of the movie is indicated for each frame. Before $t=30 \mathrm{~s}$, there is nucleation of a cation-disordered region at the location labeled by the red arrow. The SI phase (marked by green outlines) grows across the HNP over time. b FFT of the VO region (top) and SI region (bottom) of the HNP, obtained from the $t=35 \mathrm{~s}$ frame shown in Fig. 1a. The VO phase shows characteristic vacancy super-lattice spots $(d \sim 0.68 \mathrm{~nm}$, marked by blue circles) in the FFT, which are absent for the SI phase. c HRTEM snapshots of an HNP before, around, and after the point of nucleation of the order-disorder transition. Corresponding FFTs are shown in Supplementary Fig. 3. This evolution is shown for three different HNPs (one per row). The nucleation site, where vacancy-ordering is first observed to be lost, is marked by a red arrow in each case. This site was identified on the basis of a difference in the local lattice fringe pattern in the before and after frames. It is found that nucleation occurs at a vertex site at the intersection of (111) $)_{c}$ and $(100)_{c}$ surface facets. These surface facets (labeled in the leftmost image of each row) were assigned on the basis of FFT analysis of the HRTEM images of individual HNPs, as described in Supplementary Fig. 4 . Scale bars in images are 5 nm in length

We studied the influence of electron dose rate and accelerating voltage on the phase transition speed (Fig. 2b). The transition speed was defined as the inverse of the time required for the completion of a phase transition in an HNP. The higher the electron dose rate, at a constant accelerating voltage, the higher was the speed of the transition. At a similar electron dose rate, the transition speed was lower for higher accelerating voltages. These trends indicate that inelastic scattering processes are responsible for the electron beam-induced phase transition. We can further rule out that the phase transition is induced by the knock-out displacement of $\mathrm{Cu}$ because this process requires a critical accelerating voltage of $420 \mathrm{kV}^{30}$, which is higher than the accelerating voltages used in our studies. Even though electrostatic charging of the HNP cannot be ruled out, these results, combined with the estimated local temperature of our electron beam irradiation, indicate that beam-induced heating is the primary means for inducing the VO-to-SI phase transition. Consistent with this interpretation, the higher 

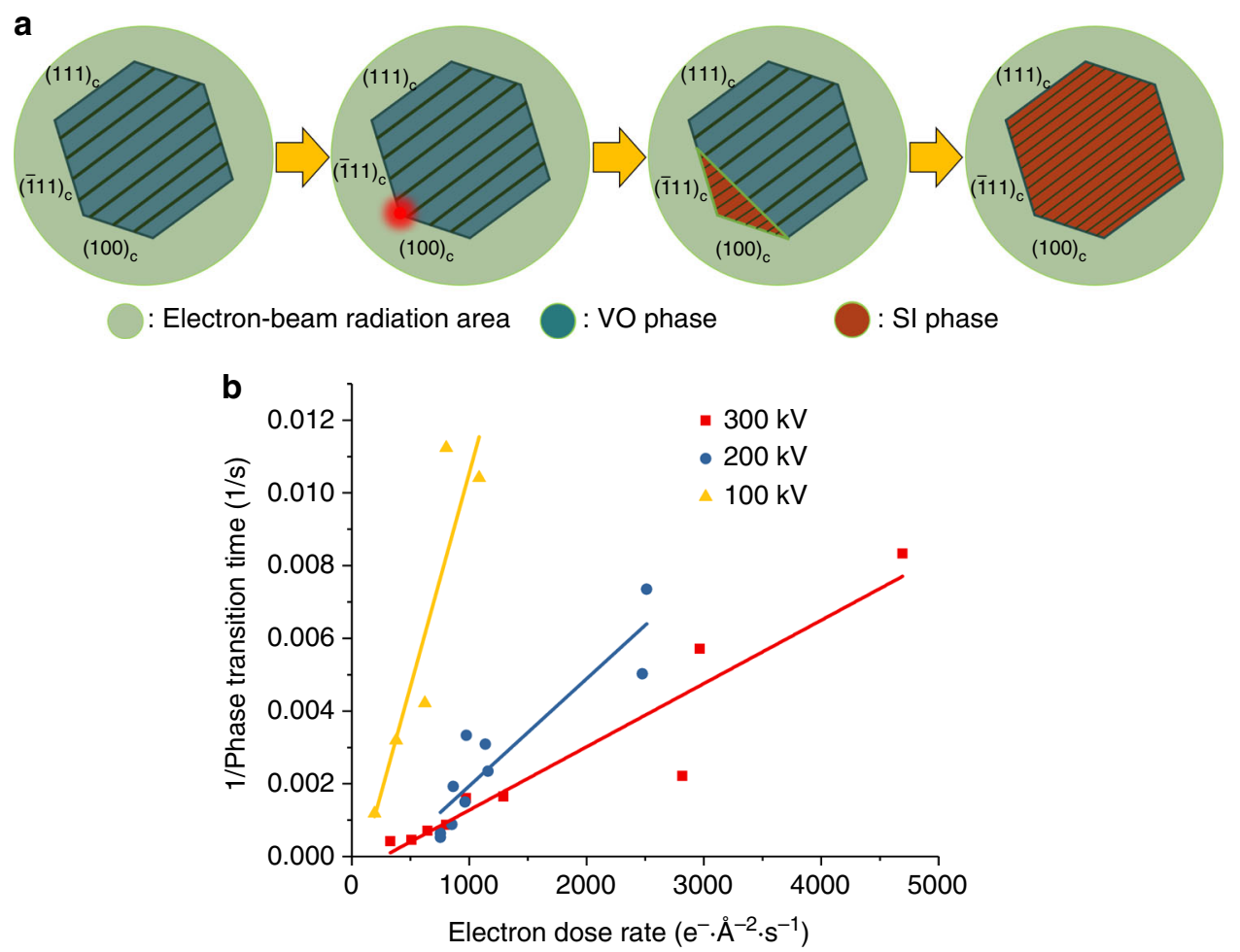

Fig. 2 Spatiotemporal progression of the order-disorder transition. a Schematic of the electron-beam induced transition of the $\mathrm{Cu}_{2-x} \mathrm{Se} H N P s$ from the $\mathrm{VO}$ phase to the SI phase. $\mathbf{b}$ The speed of the phase transition as a function of the electron dose rate at three different accelerating voltages. The phase transition speed is defined by the inverse of the time it takes from the start of the acquisition for the entire $\mathrm{Cu}_{2-x} \mathrm{Se} \mathrm{HNP}$ to transition into the SI phase. Each data-point is obtained from a separate single HNP subject to electron-beam irradiation of a fixed electron dose rate at a fixed accelerating voltage. Solid lines are meant to guide the eye

the electron dose rate, the higher is the temperature reached within the beam-irradiated HNP (Supplementary Note), and faster is the transition. In effect, the electron beam parameters can be employed as handled for controlling the phase transition kinetics.

The beam-induced phase transition was also found to be reversible (Supplementary Fig. 5a). When an HNP that had fully transitioned into the SI phase was allowed to stand in the absence of electron beam irradiation, the HNP reverted to the VO phase. Thus, it appears that the HNP does not suffer any irreversible damage or structural changes due to electron beam irradiation. In the absence of beam irradiation, the HNP presumably returns to a temperature below the phase transition temperature, resulting in the recovery of the low-temperature VO phase. We also observed that if the electron beam irradiation was interrupted while an HNP was in the midst of a phase transition, the HNP began to revert back to the VO phase (Supplementary Fig. 5b). Thus, beam-induced heating appears to be needed not only for initiating the transition but also for maintaining the higher temperature needed for the SI phase to extend across the entire HNP. Thus, it is possible to control the phase of the HNP by modulation of the electron-beam irradiation, with possible applications in electron-beam writing of information at highdensities. Information encoded in the form of the phase can be read out at low electron beam dose rates: for instance, at a dose rate below $147 \mathrm{e}^{-} \AA^{-2} \mathrm{~s}^{-1}$ at $300 \mathrm{kV}$, no phase transition was observed for at least $30 \mathrm{~min}$ and the HNP remained in the VO phase.

Kinetics and dynamics of the phase transition. In addition to the identification of the atomic site of nucleation, the structural dynamics of the phase transition was also revealed by in-situ HRTEM.
As described in a previous section, the SI phase of $\mathrm{Cu}_{2-x}$ Se lacks the ordered super-lattice arrangement of the $\mathrm{Cu}^{+}$sub-lattice that the VO phase exhibits. There is one additional structural difference: the overall unit cell is known to have a smaller volume ${ }^{26}$ in the SI phase as compared to the VO phase. We observed a signature of this lattice volume difference in the $\mathrm{Se}^{2-}-\mathrm{Se}^{2-}$ interplanar spacing along $[111]_{c}$ measured from FFTs (Fig. 3c) of HRTEM snapshots. The starting HNPs exhibit a $\mathrm{Se}^{2-}-\mathrm{Se}^{2-}$ interplanar spacing of $\sim 0.34 \mathrm{~nm}$ in the VO phase; whereas posttransition, the HNPs show a moderately compressed lattice with a $\mathrm{Se}^{2-}-\mathrm{Se}^{2-}$ interlayer spacing of $\sim 0.33 \mathrm{~nm}$, which is $\sim 3 \%$ smaller than that in the VO phase.

From HRTEM movies of single HNPs, we were able to monitor the real-time evolution of the aforementioned structural features as the HNP transitioned from the VO phase to the SI phase. For this purpose, the HRTEM movies were subject to a frame-byframe FFT analysis, which yielded pair distribution functions (PDFs) as a function of time (Fig. 3). Four different sections of an HNP (Fig. 3a) were separately analyzed to gain a spatially resolved perspective.

As the $\mathrm{Cu}^{+}$sub-lattice becomes locally disordered in a section of the HNP, the reciprocal-lattice peak near $1.5 \mathrm{~nm}^{-1}$, which corresponds to $\mathrm{Cu}$-vacancy ordering, decreases in intensity until the peak finally disappears when the transition to the disordered phase is complete in that section (Fig. 3b). Alongside, the time evolution of the $\mathrm{Se}^{2-}$ sub-lattice is manifested in the reciprocallattice peak near $3 \mathrm{~nm}^{-1}$, which corresponds to the $\mathrm{Se}^{2-}-\mathrm{Se}^{2-}$ interplanar spacing along $[111]_{c}$. With increasing time, the peak shifts to higher reciprocal lattice spacing $\left(d^{-1}\right)$ or smaller $d$ spacing, indicating a compression of the lattice. What is most notable is that this lattice compression begins to occur prior to the lattice fully adopting the SI phase. This trend is observed for 


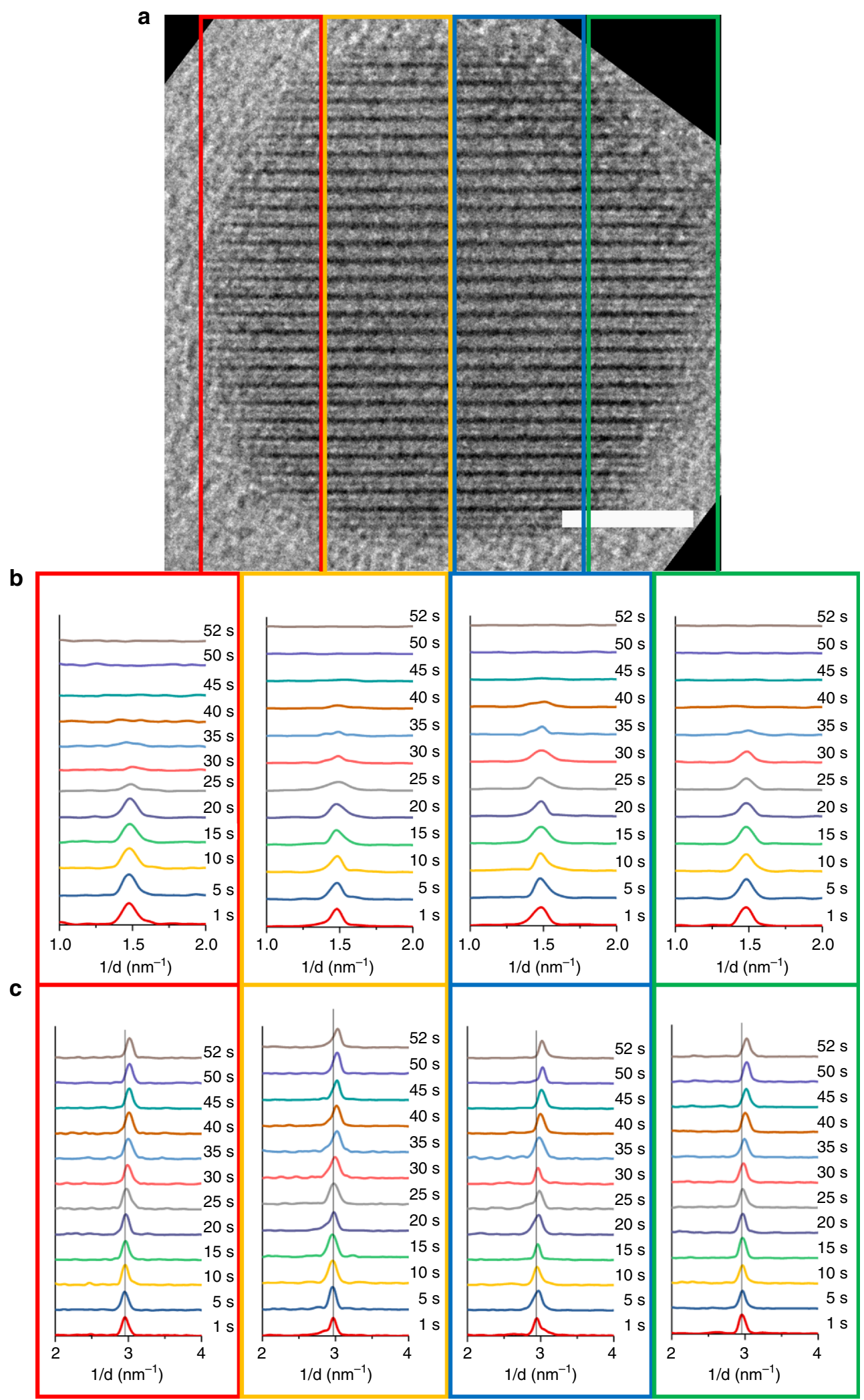

all four regions of the HNP shown in Fig. 3 and in 9 additional HNPs presented in Supplementary Fig. 6. This observation makes evident a correlated re-organization of the anionic and the cationic sub-lattices of $\mathrm{Cu}_{2-\mathrm{x}} \mathrm{Se}$ in the order-disorder transition.

Such a correlated structural re-organization is reproduced in density functional theory (DFT) computations (Fig. 4). Energy relaxation of a $\mathrm{Cu}_{2-\mathrm{x}} \mathrm{Se}$ unit cell bearing a $\mathrm{VO}$ structure resulted in only minor displacement of the $\mathrm{Se}^{2-}$, whereas the $\mathrm{Cu}$-vacancy ordering was fully preserved. However, a $\mathrm{Cu}_{2-\mathrm{x}} \mathrm{Se}$ unit cell with a lattice compressed by $3.9 \%$ isotropically (including along [111 $]_{c}$ ) lost its ordered $\mathrm{Cu}$-vacancy arrangement upon energy relaxation. This observation is consistent with a previous postulate that compressive strain stabilizes $\mathrm{Cu}^{+}$occupancy of otherwise higherenergy octahedral sites ${ }^{23,34}$. When $\mathrm{Cu}^{+}$gain access to these new 
Fig. $3 \mathrm{Se}^{2-}$ sub-lattice undergoes compression alongside the loss of vacancy ordering. a HRTEM image, with a 5-nm scale bar, of an HNP oriented with the $[111]_{c}$ direction aligned vertically. This is the same HNP as the one shown in Fig. 1a. The oriented HNP lattice is sub-divided into four distinct regions, which are outlined by the four colored boxes. $\mathbf{b}$ The time-evolution of the PDFs in the $d^{-1}=1-2 \mathrm{~nm}^{-1}$ range for each of the four regions in the corresponding column. Every PDF was normalized by dividing by the maximum of the PDF with the highest intensity peak in that series. $\mathbf{c}$ The time-evolution of the PDFs in the $d^{-1}=2-4 \mathrm{~nm}^{-1}$ range for each of the four regions in the corresponding column. Every PDF was separately normalized from 0 to 1 . The PDFs are stacked vertically as a function of the time-point in the movie. The vertical line is aligned with the maximum of the reciprocal-lattice peak at the starting time-point. For the $1 \mathrm{~s}$ and $52 \mathrm{~s}$ frames, the reciprocal-lattice peak around $3 \mathrm{~nm}^{-1}$, which corresponds to the $\mathrm{Se}^{2-}-\mathrm{Se}^{2-}$ interplanar spacing along [111] $]_{\mathrm{C}}$ was fitted with a Gaussian function to yield the $d$-spacing, which was found to be $0.34 \mathrm{~nm}(\mathrm{SD}=0.34 \%)$ and $0.33 \mathrm{~nm}(\mathrm{SD}=0.10 \%)$, respectively. The reported $d$-spacing is an average over the four regions and the \% standard deviation is indicated in the parentheses. From the PDFs, it is seen that the $\mathrm{Se}^{2-}-\mathrm{Se}^{2-}$ peak shifts to smaller $d$-spacings alongside the loss of the Cu-vacancy super-lattice peak around $1.5 \mathrm{~nm}^{-1}$. The same behavior is shown by all 10 HNPs arbitrarily selected for this analysis from the total available sample set of 15 HNPs (see Supplementary Fig. 6)

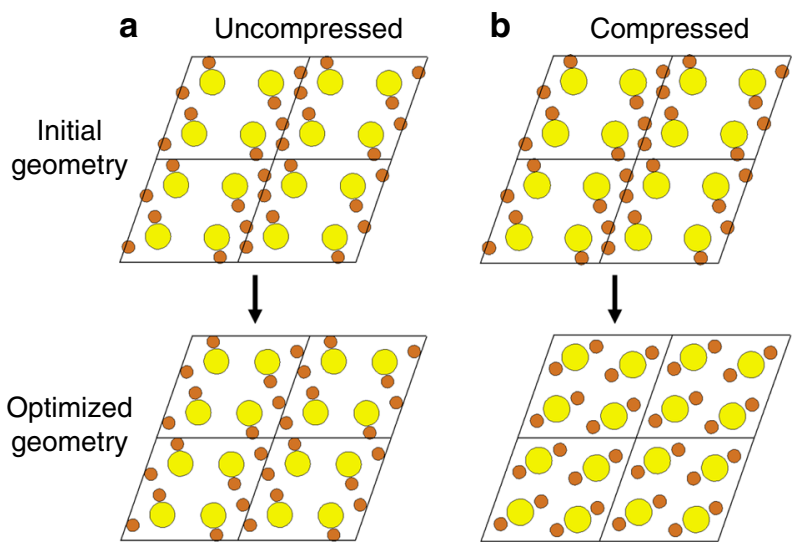

Fig. 4 DFT investigation of the correlation between lattice strain and cation disorder. $\mathbf{a}$ In the absence of any strain, a $\mathrm{Cu}_{8} \mathrm{Se}_{4}$ unit cell maintains its $\mathrm{Cu}$ vacancy order upon geometry optimization. b In contrast, a $\mathrm{Cu}_{8} \mathrm{Se}_{4}$ unit cell, subject to a $3.9 \%$ compressive strain, loses Cu-vacancy order upon geometry optimization. $\mathrm{Se}^{2-}$ is shown in yellow and $\mathrm{Cu}^{+}$in orange. Note that $2 \times 2 \times 1$ supercells are shown for easier visualization of the vacancy ordering. The black arrow signifies the geometry optimization procedure. The unit cell parameters for the two cases are described in the methods

interstitial (octahedral ${ }^{24,25}$ or other nearby ${ }^{35}$ ) sites, the $\mathrm{Cu}^{+}$sublattice becomes mobile and the $\mathrm{Cu}$-vacancy ordering is lost.

In conclusion, in-situ HRTEM imaging allowed atomic-level monitoring of an order-disorder transition in nanoparticles of $\mathrm{Cu}_{2-\mathrm{x}} \mathrm{Se}$, a model super-ionic conductor. We were able to resolve super-lattice ordered and cation-disordered domains within individual HNPs and follow the time-evolution of these domains over the course of the transition. Enabled by the atomic resolution, we identified the site where cation disorder is nucleated. The nucleation site is found to be located on the HNP surface, specifically at vertices where the atomic coordination is significantly lower than in the bulk due to the confluence of point defects (Cu-vacancies) with surface atoms. Secondly, the HRTEM movies reveal that the loss of Cu-vacancy ordering is accompanied by a lattice compression of the $\mathrm{Se}^{2-}$ cage. Such correlated motions exemplify the mechanistic richness of phase transitions. More specifically, we gain a deeper understanding of the cation disorder in $\mathrm{Cu}_{2-\mathrm{x}} \mathrm{Se}$, the control of which would enable high-performance solid-electrolytes ${ }^{18,36}$.

\section{Methods}

$\mathbf{C u}_{2-\mathbf{x}}$ Se HNP synthesis. $\mathrm{Cu}_{2-\mathrm{x}} \mathrm{Se}$ HNPs were synthesized on the basis of a published procedure ${ }^{37}$ with some modification. In a $50 \mathrm{~mL}$, three-necked roundbottom flask, $2 \mathrm{mmol}(0.198 \mathrm{~g})$ of anhydrous $\mathrm{CuCl}$ was dissolved in a solvent mixture of $10 \mathrm{~mL}$ of oleylamine and $10 \mathrm{~mL}$ of 1 -octadecene (ODE). The $\mathrm{CuCl}$ solution was heated to a temperature of $80^{\circ} \mathrm{C}$ and subject to vacuum degassing for $2 \mathrm{~h}$. The temperature was then raised to $330^{\circ} \mathrm{C}$ under Ar flow. A fresh Se-ODE solution was prepared in the glove box by dissolving $1 \mathrm{mmol}(0.078 \mathrm{~g})$ of Se in $5 \mathrm{~mL}$ of ODE and heating the mixture to $200^{\circ} \mathrm{C}$ for 20 min under stirring, followed by sonication for $10 \mathrm{~min}$ until the Se dissolved. At $330^{\circ} \mathrm{C}$ under $\mathrm{Ar}$ flow, the Se-ODE solution was rapidly injected into the $\mathrm{CuCl}$ solution in the reaction flask. Upon addition of the Se-ODE solution, the temperature dropped. Following the recovery of the temperature to $310^{\circ} \mathrm{C}$, the reaction was allowed to proceed for $15 \mathrm{~min}$. After this period, the reaction mixture was allowed to cool and then $10 \mathrm{~mL}$ of toluene was added to it. The reaction mixture was transferred to the glove box for oxygen and moisture-free processing. The reaction mixture was diluted with $5 \mathrm{~mL}$ of ethanol and subject to centrifugation at $20 \times g$ for $5 \mathrm{~min}$, for removal of unreacted precursors, which stay in the resulting supernatant. The precipitate containing $\mathrm{Cu}_{2-x} \mathrm{Se}$ nanoparticles and large $\mathrm{Cu}_{2-\mathrm{X}} \mathrm{Se}$ nanoplatelets, capped with oleylamine ligands, was dispersed in $5 \mathrm{~mL}$ of toluene and subject to another round of purification by the addition of $1 \mathrm{~mL}$ of ethanol and low-speed centrifugation at $20 \times g$ for $5 \mathrm{~min}$. The supernatant from this procedure, which is relatively purified of large nanoplatelets, was subject to multiple cycles of centrifugation. The final precipitate of this procedure containing the $\mathrm{Cu}_{2-x} \mathrm{Se}$ HNPs was stored in the glovebox for further use. The HNPs were dispersed in toluene for further characterization.

In-situ HRTEM imaging and analysis. For HRTEM imaging, samples were prepared by casting a few drops of the HNP colloid on a TEM substrate consisting of an ultrathin carbon on a lacey carbon film supported by a 400 mesh Cu grid (Ted Pella Co.). HRTEM imaging was performed on a Hitachi 9500 instrument equipped with an Orius charge-coupled device (CCD) camera. Unless otherwise noted, HRTEM was performed with an accelerating voltage of $300 \mathrm{kV}$ and an electron beam current of 2 or $6 \mu \mathrm{A}$. Low-magnification images were acquired for surveying nanoparticle morphology, which show that the nanoparticles have a hexagonal shape (Supplementary Fig. 1).

For in-situ monitoring of the order-disorder transition within individual HNPs, continuous higher magnification HRTEM imaging of a fixed region containing well-isolated HNP or HNPs was performed. Movies were acquired at a rate of 1 frame $\mathrm{s}^{-1}$. The electron beam irradiation was kept uninterrupted during the course of the phase transition. HRTEM movie frames and their FFTs were analyzed using the software ImageJ to determine the time-evolution of the VO and SI phase regions within the HNP.

A separate set of studies was conducted to examine how the electron dose rate and accelerating voltage influence the ability to induce the phase transition and the effect of these parameters on the time required for completion of a phase transition. In each study, an individual HNP was subject to electron beam irradiation of a fixed electron beam dose rate at a fixed accelerating voltage and a movie was recorded. Several such individual HNP experiments were conducted at three different accelerating voltages $(100,200$, and $300 \mathrm{kV})$ and several different electron dose rates. The latter was changed by variation of the condenser aperture and/or the brightness variable. Electron dose rate (EDR) was determined from the recorded exposure density at the phosphorescent screen as follows:

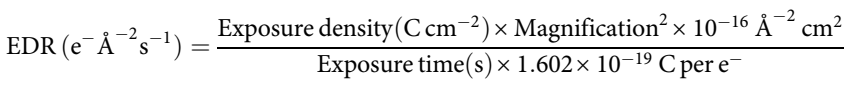

Note that if an HNP did not show any sign of nucleation in a 30-min period from the start of the acquisition, then the acquisition was stopped and the HNP was recorded to undergo no transition at those conditions. For instance, at $300 \mathrm{kV}$ at an electron beam dose rate below $147 \mathrm{e}^{-} \AA^{-2} \mathrm{~s}^{-1}$, no nucleation of the SI phase was observed in the 30-min period. On the other hand, if nucleation was observed before the 30-min time-point, the electron beam irradiation was continued without interruption until the phase transition was complete. Movies from these latter HNPs comprise the data plotted in Fig. $2 b$.

Analysis of phase-transition dynamics. HRTEM movie frames were re-oriented such that the $[111]_{c}$ direction of the HNP lattice was aligned along the line-profile axis. It must be noted that in the course of continuous imaging, there is thermal drift of the region being imaged. We corrected movie frames for this drift using the ImageJ Java plugin "NMS_fixTranslation_ver1.ijm"2. This plugin requires that the 
same landmark spot be manually identified in each movie frame to serve as the reference point for drift correction. The rest of the correction is automated by the algorithm. The manual reference point selection can introduce an error. This error is estimated to be a few pixels, which is considerably smaller than the hundreds-ofpixels dimensions of the regions selected for the section-by-section FFT analysis. Following drift correction, the movie was cropped around the HNP of interest and the HNP was divided into four sections as shown in Fig. 3. For each of these four regions, a line profile across the lattice fringe pattern along $[111]_{\mathrm{c}}$ was obtained using the open-source macro "StackProfileData_code.txt"38. One-dimensional FFTs of these line profiles were obtained using OriginPro program. The timeevolution of these PDFs formed the basis of the kinetic analyses described in Fig. 3 and Supplementary Fig. 6.

DFT simulations. Plane-wave DFT simulations of $\mathrm{Cu}_{8} \mathrm{Se}_{4}$ unit cells were performed on the Quantum Espresso suite ${ }^{39}$ using norm-conserving Se.pbe-hgh.UPF and Cu.pbe-d-hgh.UPF pseudopotentials, the Perdew-Burke-Ernzerhof functional $^{40}$, a kinetic energy cut-off of 200 Ry for wavefunctions, a 0.019 Ry smearing of electronic occupations, and automatically generated $6 \times 8 \times 6$ Monkhorst-Pack k-point grids. The geometry of the uncompressed cell (Fig. 4) was adapted from a reported $\beta-\mathrm{Cu}_{2} \mathrm{Se}$ geometry ${ }^{41}$ and had unit cell parameters of $a=7.453 \AA, b=$ $44.322 \AA, c=6.880 \AA, \alpha=90.00^{\circ}, \beta=70.62^{\circ}$, and $\gamma=90.00^{\circ}$. The $\mathrm{Se}^{2-}-\mathrm{Se}^{2-}$ interlayer spacing along $\langle 111\rangle_{\text {c }}$ was $3.52 \AA$. To introduce compressive strain, the lattice constants were decreased by $3.9 \%$. The compressed unit cell had parameters of $a=7.162 \AA, b=4.153 \AA, c=6.612 \AA, \alpha=90.00^{\circ}, \beta=70.62^{\circ}$, and $\gamma=90.00^{\circ}$, but the atomic coordinates, expressed in crystal vector units, were the same as those in the uncompressed unit cell. The $\mathrm{Se}^{2-}-\mathrm{Se}^{2-}$ interlayer spacing along [111] $]_{\mathrm{c}}$ was $3.38 \AA$ in the compressed geometry. The uncompressed and compressed unit cells were each subject to geometry optimization. The optimization procedure was accomplished by constraining $\mathrm{Se}^{2-}$ and allowing $\mathrm{Cu}^{+}$to relax and then allowing both $\mathrm{Se}^{2-}$ and $\mathrm{Cu}^{+}$to relax without constraints. The $\mathrm{Se}^{2-}-\mathrm{Se}^{2-}$ interlayer spacing along $[111]_{\mathrm{c}}$ in the geometry-optimized cells (3.51 $\AA$ and $3.37 \AA$ for uncompressed and compressed cases, respectively) was not significantly altered by geometry optimization.

\section{Data availability}

All raw images and source data are available from the authors upon reasonable request.

Received: 21 September 2018 Accepted: 13 March 2019

Published online: 03 April 2019

\section{References}

1. Park, J. H. et al. Control of electron beam-induced Au nanocrystal growth kinetics through solution chemistry. Nano Lett. 15, 5314-5320 (2015).

2. Schneider, N. M., Park, J. H., Norton, M. M., Ross, F. M. \& Bau, H. H. Automated analysis of evolving interfaces during in situ electron microscopy. Adv. Struct. Chem. Imag. 2, 2 (2017).

3. Zheng, $\mathrm{H}$. et al. Observation of transient structural-transformation dynamics in a $\mathrm{Cu}_{2} \mathrm{~S}$ nanorod. Science 333, 206-209 (2011).

4. Zheng, $\mathrm{H}$. et al. Controlling electron beam-induced structure modifications and cation exchange in cadmium sulfide-copper sulfide heterostructured nanorods. Ultramicroscopy 134, 207-213 (2013).

5. Moghadam, M. M. et al. In situ crystallization and morphological evolution in multicomponent indium oxide thin films. Cryst. Growth Des. 17, 1396-1403 (2017).

6. Panciera, F. et al. Controlling nanowire growth through electric field-induced deformation of the catalyst droplet. Nat. Commun. 7, 12271 (2016).

7. Baldi, A., Narayan, T. C., Koh, A. L. \& Dionne, J. A. In situ detection of hydrogen-induced phase transitions in individual palladium nanocrystals. Nat. Mater. 13, 1143-1148 (2014).

8. Boyce, J. B. \& Huberman, B. A. Superionic conductors: transitions, structures. Dyn. Phys. Rep. 51, 189-265 (1979).

9. Hull, S., Keen, D. A., Hayes, W. \& Gardner, N. J. G. Superionic behaviour in copper (I) iodide at elevated pressures and temperatures. J. Phys. Condens. Matter 10, 10941 (1998).

10. Li, J., Ma, C., Chi, M., Liang, C. \& Dudney, N. J. Solid electrolyte: the key for high-voltage lithium batteries. Adv. Energy Mater. 5, 1401408 (2015).

11. Quartarone, E. \& Mustarelli, P. Electrolytes for solid-state lithium rechargeable batteries: recent advances and perspectives. Chem. Soc. Rev. 40, 2525-2540 (2011).

12. Kamaya, N. et al. A lithium superionic conductor. Nat. Mater. 10, 682-686 (2011).

13. Terabe, K., Hasegawa, T., Nakayama, T. \& Aono, M. Quantized conductance atomic switch. Nature 433, 47-50 (2005)

14. Hu, T., Wittenberg, J. S. \& Lindenberg, A. M. Room-temperature stabilization of nanoscale superionic $\mathrm{Ag}_{2} \mathrm{Se}$. Nanotechnology 25, 415705 (2014).
15. Goodenough, J. B. \& Singh, P. Review-solid electrolytes in rechargeable electrochemical cells. J. Electrochem. Soc. 162, A2387-A2392 (2015).

16. Liu, H. et al. Copper ion liquid-like thermoelectrics. Nat. Mater. 11, 422-425 (2012).

17. Banerjee, P. \& Jain, P. K. Lithiation of copper selenide nanocrystals. Angew. Chem. Int. Ed. 57, 9315-9319 (2018).

18. Takahashi, T., Yamamoto, O., Matsuyama, F. \& Noda, Y. Ionic conductivity and coulometric titration of copper selenide. J. Solid State Chem. 16, 35-39 (1976).

19. Danilkin, S. A. et al. Crystal structure and lattice dynamics of superionic copper selenide $\mathrm{Cu}_{2-\delta}$ Se. J. Alloy. Compd. 1-2, 57-61 (2003).

20. Brown, D. R. et al. Phase transition enhanced thermoelectric figure-of-merit in copper chalcogenides. APL Mater. 1, 052107 (2013).

21. Kashida, S. \& Akai, J. X-ray diffraction and electron microscopy studies of the room-temperature structure of $\mathrm{Cu}_{2} \mathrm{Se}$. J. Phys. C Solid State Phys. 21, 5329 (1988).

22. Heo, J., Dumett Torres, D. \& Jain, P. K. Unconventional long-range cation ordering in copper selenide nanocrystals. Chem. Mater. 31, 68-72 (2019).

23. White, S. L., Banerjee, P. \& Jain, P. K. Liquid-like cationic sub-lattice in copper selenide clusters. Nat. Commun. 8, 14514 (2017)

24. Yamamoto, K. \& Kashida, S. X-ray study of the average structures of $\mathrm{Cu}_{2} \mathrm{Se}$ and $\mathrm{Cu}_{1.8} \mathrm{~S}$ in the room temperature and the high temperature phases. J. Solid State Chem. 93, 202-211 (1991).

25. Yamamoto, K. \& Kashida, S. X-ray study of the cation distribution in $\mathrm{Cu}_{2} \mathrm{Se}$, $\mathrm{Cu}_{1.8} \mathrm{Se}$ and $\mathrm{Cu}_{1.8} \mathrm{~S}$; analysis by the maximum entropy method. Solid State Ion. 48, 241-248 (1991).

26. Milat, O., Vučić, Z. \& Ruščić, B. Superstructural ordering in low-temperature phase of superionic $\mathrm{Cu}_{2} \mathrm{Se}$. Solid State Ion. 23, 37-47 (1987).

27. Vučić, Z., Milat, O., Horvatić, V. \& Ogorelec, Z. Composition-induced phasetransition splitting in cuprous selenide. Phys. Rev. B 24, 5398-5401 (1981).

28. Frangis, N., Manolikas, C. \& Amelinckx, S. Vacancy-ordered superstructures in $\mathrm{Cu}_{2}$ Se. Phys. Status Solidi A 126, 9-22 (1991).

29. Heyding, R. D. \& Murray, R. M. The crystal structures of $\mathrm{Cu}_{1.8} \mathrm{Se}, \mathrm{Cu}_{3} \mathrm{Se}_{2}$, a and $\gamma \mathrm{CuSe}, \mathrm{CuSe}_{2}$, and $\mathrm{CuSe}_{2}$ II. Can. J. Chem. 54, 841-848 (1976).

30. Egerton, R. F., Li, P. \& Malac, M. Radiation damage in the TEM and SEM. Micron 35, 399-409 (2004)

31. Sytwu, K. et al. Visualizing facet-dependent hydrogenation dynamics in individual palladium nanoparticles. Nano Lett. 18, 5357-5363 (2018).

32. Cazaux, J. Correlations between ionization radiation damage and charging effects in transmission electron microscopy. Ultramicroscopy 60, 411-425 (1995).

33. Tao, J. et al. Reversible structure manipulation by tuning carrier concentration in metastable $\mathrm{Cu}_{2}$ S. Proc. Natl Acad. Sci. USA 114, 9832-9837 (2017).

34. Dumett Torres, D. \& Jain, P. K. Strain stabilization of superionicity in copper and lithium selenides. J. Phys. Chem. Lett. 9, 1200-1205 (2018).

35. Dalgaard, K. J., Eikeland, E. Z., Sist, M. \& Iversen, B. B. Maximum entropy method visualization of disorder and ion migration in thermoelectric $\mathrm{Cu}_{2-\delta} \mathrm{Se}$. Adv. Theory Simul. 1, 1800068 (2018).

36. Wang, Y. et al. Design principles for solid-state lithium superionic conductors. Nat. Mater. 14, 1026-1031 (2015)

37. Deka, S. et al. Phosphine-free synthesis of p-type copper (I) selenide nanocrystals in hot coordinating solvents. J. Am. Chem. Soc. 132, 8912-8914 (2010).

38. Schneider, C. A., Rasband, W. S. \& Eliceiri, K. W. NIH Image to ImageJ: 25 years of image analysis. Nat. Methods 9, 671-675 (2012).

39. Giannozzi, P. et al. Quantum ESPRESSO: a modular and open-source software project for quantum simulations of materials. J. Phys. Condens. Matter 21 395502 (2009)

40. Perdew, J. P., Burke, K. \& Ernzerhof, M. Generalized gradient approximation made simple. Phys. Rev. Lett. 77, 3865-3868 (1996).

41. Chi, H. et al. Low-temperature structural and transport anomalies in $\mathrm{Cu}_{2} \mathrm{Se}$. Phys. Rev. B 89, 195209 (2014)

\section{Acknowledgements}

Funding for this work was provided by the Energy \& Biosciences Institute (EBI) through the EBI-Shell program. This work was carried out in part at the Frederick Seitz Materials Research Lab. We acknowledge the donors of the American Chemical Society Petroleum Research Fund for support of our preliminary work. The Extreme Science and Engineering Discovery Environment (XSEDE) research allocation and SEAGrid computational resources were used for calculations. We acknowledge Sudhakar Pamidighantam for his support of our computations.

\section{Author contributions}

J.H. performed all experimental studies and data-analysis, and co-wrote the manuscript D.D.T. performed DFT studies and corresponding analysis and co-wrote the manuscript P.B. performed nanoparticle synthesis. P.K.J. designed experimental and simulation studies, provided analytical methods and interpretation of results, and co-wrote the manuscript. 


\section{Additional information}

Supplementary Information accompanies this paper at https://doi.org/10.1038/s41467019-09502-5.

Competing interests: The authors declare no competing interests.

Reprints and permission information is available online at http://npg.nature.com/ reprintsandpermissions/.

Journal Peer Review Information: Nature Communications thanks Jeung Hun Park, and other anonymous reviewer(s) for their contribution to the peer review of this work

Publisher's note: Springer Nature remains neutral with regard to jurisdictional claims in published maps and institutional affiliations. (c) Open Access This article is licensed under a Creative Commons Attribution 4.0 International License, which permits use, sharing, adaptation, distribution and reproduction in any medium or format, as long as you give appropriate credit to the original author(s) and the source, provide a link to the Creative Commons license, and indicate if changes were made. The images or other third party material in this article are included in the article's Creative Commons license, unles indicated otherwise in a credit line to the material. If material is not included in the article's Creative Commons license and your intended use is not permitted by statutory regulation or exceeds the permitted use, you will need to obtain permission directly from the copyright holder. To view a copy of this license, visit http://creativecommons.org/ licenses/by/4.0/.

(C) The Author(s) 2019 